\title{
TRICHOBEZOAR VENTRICULI IN 7-YEAR OLD GIRL - CASE REPORT
}

\author{
Miodrag Stankovic ${ }^{1}$, Zlatko Djuric ${ }^{2}$, Zoran Marjanovic ${ }^{3}$, Aleksandra Stojanovic ${ }^{1}$, \\ Jelena Kostic ${ }^{1}$ \& Sandra Stankovic ${ }^{2}$ \\ ${ }^{I}$ Clinic for Mental Health Protection, Clinical Center, Nis, Serbia \\ ${ }^{2}$ Clinic for Children's Internal Diseases, Nis, Serbia \\ ${ }^{3}$ Clinic for Children's Surgery, Nis, Serbia
}

received: 22.11.2016;

revised: 28.3.2017;

accepted: 28.4.2017

$* * * * *$

\section{INTRODUCTION}

Gastric trichobezoar presents a compact mass of undigested hair inside a ventricular cavity. „Bezoar“ is the name of Arab origin and denotes an antidote, which was the result of a belief that animal stomach stone had a healing feature (Shorter 2005). Forming of a trichobezoar is a rare medical condition. In literature, the majority of presented cases are ones of female adolescents (Jain et al. 2011), there are just a few case reports of preadolescents. Forming of a trichobezoar is usually a result of hair swallowing (trichophagia). In one third of the patients with trichophagia, its result is forming of a trichobesoar (Salaam et al. 2005). Trichophagia as part of pica disorder, can be a reflection of severe neglect and lack of parental supervision (Rose et al. 2000). Depending on the different authors, it is estimated that $5-20 \%$ of people with trichophagia also have a compulsive need for pulling out their own hair (trichotillomania) (Grant \& Odlaug 2005). Trichotillomania is a psychiatric impulse control disorder (Colomb et al. 2011), and according to DSM-5 classification falls within spectrum of obsessive compulsive disorders (American Psychiatric Association 2013). Trichotillomania is usually connected to hair pulling, but eyebrow, eyelashes and pubic region hair pulling can also be present (Gawłowska-Sawosz 2016). Different authors mention other psychiatric conditions connected to forming of a trichobesoar: mental retardation, obsessive compulsive disorder, depression and anorexia nervosa (Cohen et al. 1995).

Trichobezoar can easily be diagnosed by endoscopic examination or computed tomography (CT), and the therapy almost always requires surgical removal (Mewa Kinoo \& Singh 2012). The diagnosis of trichobesoar represents an etiologic and therapeutic challenge, and requires a multidisciplinary approach (Santos et al. 2012). Therapy and prevention also involve the participation of several consultants, paediatrician, surgeon, child psychiatrist, Clinic's social worker and the Community Social Service. The aim of the case report is to show the importance of multidisciplinary medical approach and role of child psychiatrists in the therapy of trichotilomania, prevention of trichobezoar, as well as in the occurrence of relapses.

\section{CASE REPORT}

Seven-year-old girl „M“ was sent to the Children's University Clinic as an emergency pediatric case with fever, enlarged lymph nodes in the cervical region, and the palpable, painless and mobile tumoroid epigastric mass. The patient had not complained of dyspeptic disorders. During hospitalization abdominal ultrasound was performed, and it showed clearly circumscribed homogeneous echogenic mass the size of $8 \mathrm{~cm}$. Esophagus-gastro-duodenal endoscopy demonstrated the existence of trichobezoar along the whole length of the stomach with extinction in the duodenum, but not in jejunum. (Figure 1).

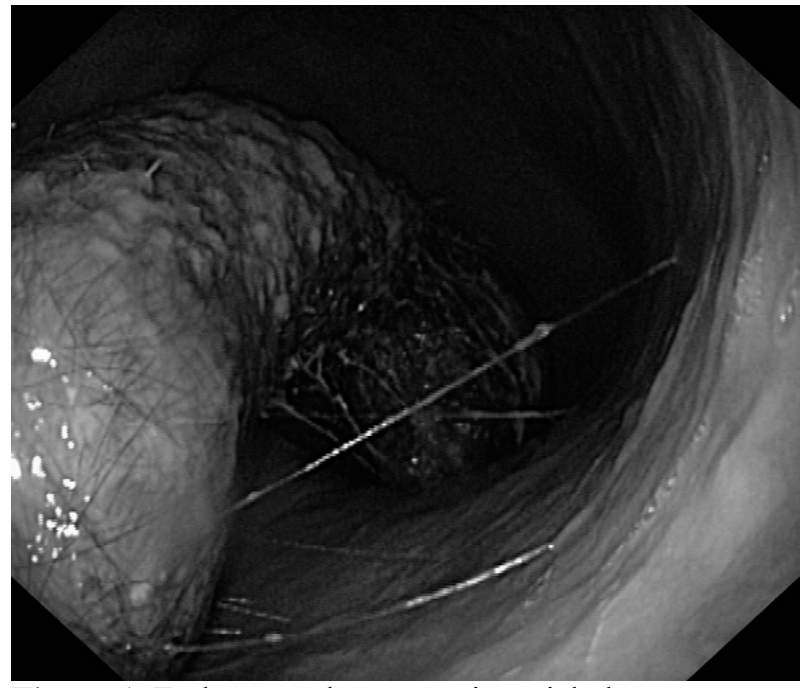

Figure 1. Endoscopy demonstrating trichobezoar extending through pylorus

Due to the size and position of trichobezoar, endoscopic extraction was not possible, so the patient was sent to pediatric surgeon who removed the trichobezoar (Figure 2).

Unexplained cause of trichobezoar and insufficiently consistent data obtained from parents, requested the inclusion of Clinic's social services, and a consultation with a child psychiatrist. It was found that patient lived with parents in a family house with poor parental supervision and poor financial income. We obtained no 


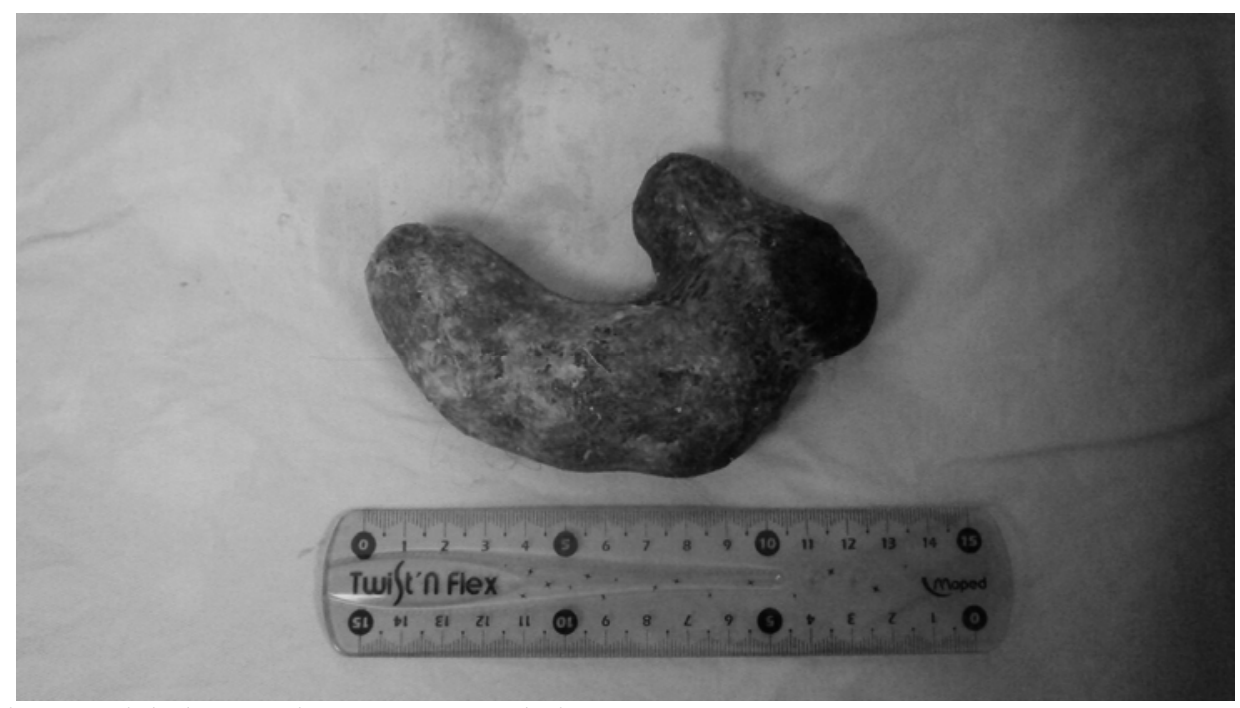

Figure 2. Gastric cast trichobezoar that was removed via gastrostomy

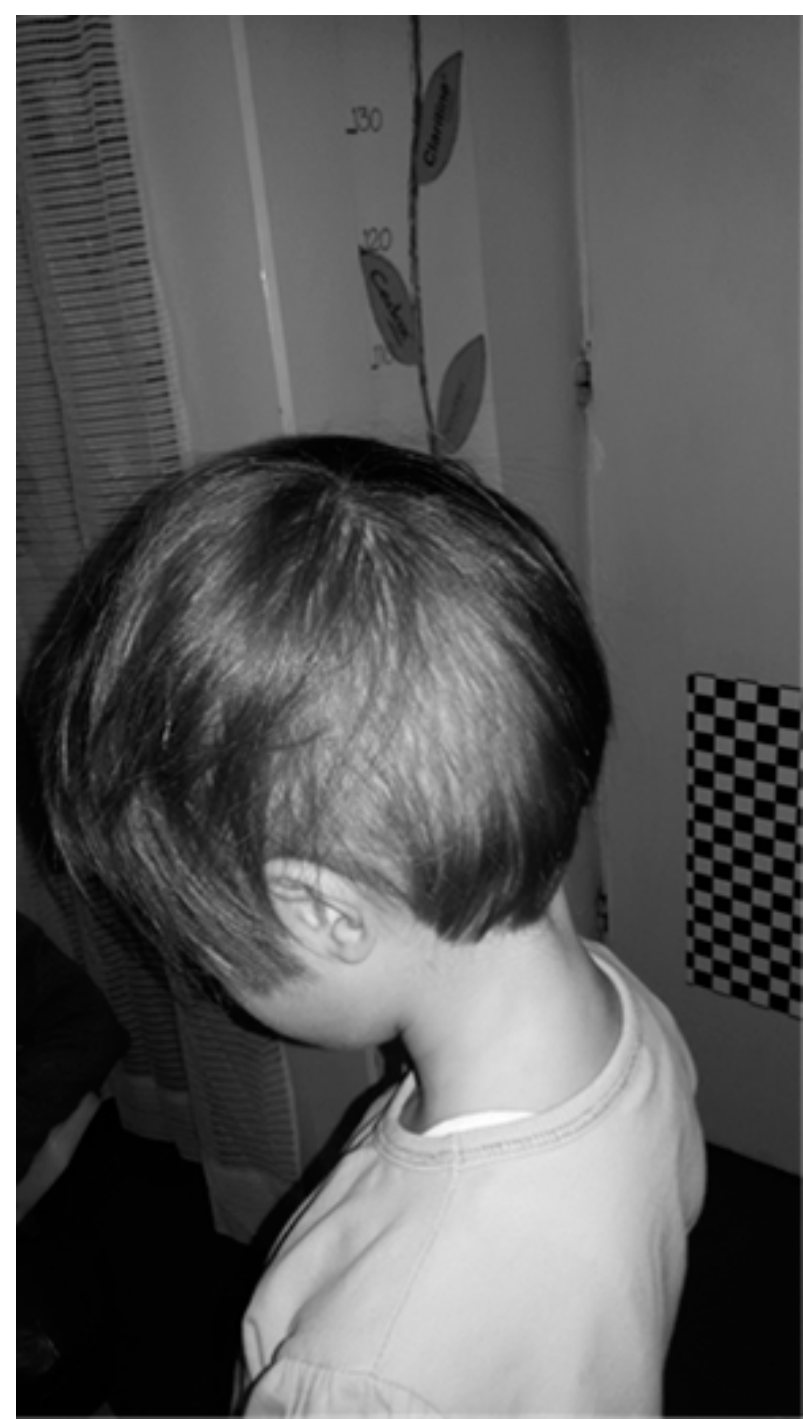

Figure 3. Sculp regions with reduced hair density (after the haircut)

informations that child had been examined by a psychologist or psychiatrist before hospitalization, nor the information that the child attended school. External inspection found the existence of circular fields of exceptionally low hair density and globally damaged hair as a result of tearing. The obtained data were that the girl had been pulling her hair on a daily basis, as well as secretly eating it. "M" said that in the beginning she had been eating hair because she had been hungry, but that it later became a habit. Scalp regions with a very low density of hair were visible (Figure 3 ). The patient was discharged from Children's surgical clinic for further psychiatric assesment and family counseling at Child Psychiatry Department.

\section{Excerpt from the life history}

Psychomotor development possibly mildly slowed (started to walk at 16 months, first sentences when she was 3 year old), without the existence of chronic physical diseases, injuries and surgical interventions, as well as allergic reactions. She did not go to school and had periods of defiant and oppositional behavior in relation to the mother whose upbringing style was largely based on the warnings, criticism and physical punishment.

\section{Excerpt from the family history}

" $\mathrm{M}$ " came from a family where the parents were unemployed and they were receiving financial support from their parents and social services. The father completed a high school, and during the last two years he took care of his parents who were immobile. The mother finished elementary school, mainly taking care of the two children ("M" is two years older than brother), but the father thought that sometimes she had not have enough control over the children and that the relationship with the girl was burdened by conflicts. Family functioning was impaired with indirect communication, avoiding conflicts, poor emotional closeness, the adaptation rigidity and passive-dependent behavior patterns. Parents denied psychiatric disorders in their families of origin. 


\section{Initial somatic and psychiatric status assesment}

During the assessment, "M" was conscious and oriented in all modalities. Walking was difficult because of the previous surgery. Nevertheless, the verbal contact was easily established, as well as eye contact. The speech was appropriate for the age. Attention was hypervigilant, with poor tenacity, estimated as untrained in relation to the age, but without psychopathological quality. A coherent thinking process, with no disorders in the content part of the opinion. Intellectual functioning was below average. Memory function preserved. The girl did not show symptoms of depressive or psychotic disorders. She did not have suicidal ideas and intentions either then or in the past. She showed parts of the head that had very little hair, due to hair pulling, and said that she had eaten it, hiding from the mother. She did not eat other non-nutritive substances.

EEG findings showed no abnormalities. Psychological testing using the Wechsler Intelligence Scale for Children (WISC) was performed. The results were: verbal IQ-66, manipulative IQ-67, overall IQ-66, SQ72. Findings showed the overall functionality of the child on a level of mild intellectual disability with educational neglect.

\section{Therapy}

The therapy was conducted in the form of four sessions of family counseling and behavior cognitive therapy, followed by three evaluation session after eight weeks, sixteen weeks and one year from initial treatment. Behavior therapy consisted in increasing awareness, stimulus control, reinforcement for avoiding touching and pulling hair. Advising parents was focused on methods of reinforcement and extension of the interval of the target behavior. In order to facilitate the positive and negative consequences, the girl willingly accepted shortening her hair.

\section{Follow up}

During the evaluation of psychosocial and psychotherapeutic treatment and monitoring the performance of parenting by social services during the one year time there were no relapse. Family reestablished social contacts, and the girl started regular education with good adaptation to staying at school and peer relationships. A complete recovery from trichotillomania and manifestation of other impulse control disorders and behavioral abnormalities were established.

\section{DISCUSSION}

In the literature, the majority of cases described trichobezoar appearance in adolescents, but rarely in preadolescent girl as we did. After diagnosing trichobezoar and surgical removal, causes of trichophagia were examined. Laboratory-biochemical analysis, neurological examination and EEG findings excluded the possible "organic" basis of the abnormal behaviour. There were no indications for magnetic resonance imaging (NMR). According other informations and clinical findings, we concluded that forming of a trichobezoar was closely linked to trichotillomania. Diagnosis was established according to ICD-10 and DSM-5 criteria. Girl recurrently pulled her hair, with failure to resist an impulse even on parent's suggestions, which resulted in hair loss, causing significant impairment in social functioning. Other psychiatric disorders such as pica, depression, psychosis, classic obsessive-compulsive disorder (OCD) and body dysmorphic disorder were also excluded. The clinical presentation of our patient had a typical evolution: trichotillomania-trihophagiatrichobezoar.

We believe that trichotillomania, as a disorder of impulse control according ICD-10, (but also an OCD spectrum disorder, according DSM-5), developed in the setting of poor parental supervision, educational neglect and mild learning disability. In the circumstances of nonstimulative family enviroment, the girl developed self-stimulative and self-sustaining patterns of behavior. This is confimed by the fact that the symptoms had been notably reduced in the course of several weeks after finishing family counseling and psychotherapy, and have not occurred in one year follow-up.

The symptoms could be explained as an instrument of active avoiding of aversive consequences of criticism and hostility of the mother keeping in mind the egodystonic nature of the conduct, accompanied by shame, guilt and denial. Impulse control disorder gets close to compulsive behavior, or to the impulsive-compulsive behavior model (Hollander 2007), and also conceptualized as behavioral addiction because of simultaines to drug addiction (Karim \& Chaudhri 2012).

Although this is a rare medical condition, the initial therapeutic protocol is complex because it generally involves the surgical procedure, with a high rate of mortality and complications associated with the existence of trichobezoar (gastric obstruction, malabsorption, bile duct obstruction, protein losing enteropathy, pancreatitis, and death) (Salaam et al. 2005).

The significance of this paper is to emphasize the need for a multidisciplinary assesment, therapy and prevention of the trichobezoar occurence. Delays in diagnosis can cause serious, even life-threatening complications. Psychiatric diagnosis of trichotillomania, is also a potential indication for detecting trichophagia as a direct cause of trichobezoar. The current recommendations are that the existence of trichobezoar should be considered in the differential diagnosis for all young women with nonspecific epigastric pain, increased fatigue, weight loss and existence of epigastric tumor mass (Altonbary \& Bahgat 2015). We believe that it is justified that this recommendations should also be applied to preadolescent females with signs of trichotillomania and poor parental supervision. 


\section{Acknowledgements: None.}

Conflict of interest: None to declare.

\section{Contribution of individual authors:}

Miodrag Stankovic: design of the case presentation, writing the article, literature searches and analyses;

Zlatko Djuric: obtaining figures, literature searches and analyses;

Zoran Marjanovic: obtaining figures, literature searches and analyses;

Aleksandra Stojanovic: writing the article, literature searches and analyses, translation;

Jelena Kostic: literature searches and analyses;

Sandra Stankovic: literature searches and analyses.

\section{References}

1. Altonbary AY, Bahgat MH:Rapunzel syndrome. J Transl Intern Med 2015; 3:79-81.

2. American Psychiatric Association (APA): Diagnostic and Statistical Manual of Mental Disorders, Fifth Edition. Diagnostic and Statistical Manual of Mental Disorders, Fifth Edition. Arlington, VA: American Psychiatric Press, Inc 2013; 271-80.

3. Cohen LJ, Stein DJ, Simeon D, Spadaccini E, Rosen J, Aronowitz B, et al.: Clinical profile, comorbidity and treatment history in 123 hairpullers: a survey study. J Clin Psychiatr 1995; 56:319-326.

4. Colomb R, Franklin M, Grant JE, Keuthen NJ, Mansueto CS, Mouton-Odum S. et al.: Expert consensus treatment guidelines for trichotillomania, skin picking and other body-focused repetitive behaviours. 2011;

http://www.trich.org/dnld/ExpertGuidelines_000.pdf [retrieved: 06.12.2015].

5. Gawłowska-Sawosz M, Wolski M, Kaminski A et al.: Trichotillomania and trichophagia - diagnosis, treatment, prevention. The attempt to establish guidelines of treatment in Poland. Psychiatr Pol 2016; 50:127-143.

6. Grant JE \& Odlaug BL: Clinical characteristics of trichotillomania with trichophagia. Compr Psychiatry 2008; 49:579-584.

7. Hollander E, Kim S, Khanna S, Pallanti S: Obsessivecompulsive disorder and obsessive-compulsive spectrum disorders: diagnostic and dimensional issues. CNS Spectr 2007; 2(suppl 3): 12 .

8. Jain M, Solanki SL, Bhatnagar A, Jain PK: An unusual case report of Rapunzel syndrome trichobezoar in a 3year-old boy. Int J Trichology 2011; 3:102-4.

9. Karim $R \&$ Chaudhri P: Behavioral addictions: An overview. Journal of Psychoactive Drugs 2012; 44:5-17.

10. Mewa Kinoo $S$ \& Singh B: Gastric Trichobezoar: An Enduring Intrigue. Case Reports in Gastrointestinal Medicine 2012; Article ID 136963, 3 pages, 2012. doi:10.1155/2012/136963.

11. Rose EA, Porcerelli JH, Neale AV: Pica: Common but commonly missed. J Am Board Fam Pract 2000; 13:353-358.

12. Salaam K, Carr J, Grewal H, Sholevar E, Baron D: Untreated trichotillomania and trichophagia: Surgical emergency in a teenage girl. Psychosomatics 2005; 46:4.

13. Santos T, Nuno M, Joao A, et al.: Trichophagia and trichobezoar: Case report. Clin Pract Epidemiol Ment Health 2012; 8:43-45.

14. Shorter E: A historical dictionary of psychiatry. Oxford University Press: New York. 2005.

Correspondence:

Assoc. Prof. Miodrag Stankovic, MD, PhD

Clinic for Mental Health Protection, Clinical Center

Nis, Serbia

E-mail: miodrag.stankovic@medfak.nj.ac.rs 